\title{
LINEAR PROGRAMMING IN SPECTRAL ESTIMATION. APPLICATION TO ARRAY PROCESSING.
}

\author{
Jean-Jacques FUCHS \\ IRISA/Université de Rennes I \\ Campus de Beaulieu - 35042 Rennes Cedex - France
}

\begin{abstract}
We address the narrow-band source localization problem for arbitrary arrays with known geometry in the presence of arbitrary noise of unknown spatial spectral density. Very few methods are able to handle this problem. We present a very unsophisticated approach whose algorithmic part relies on a standard linear programming algorithm (such as the simplex algorithm available in any scientific program library). The computational complexity of the method is reasonable, the performances appear to be remarkable on simulations. The justification of the procedure and the asymptotic analysis is more complex and much work remains to be done.
\end{abstract}

\section{INTRODUCTION}

The source localization problem for passive arrays is mostly addressed in the case of linear arrays with equispaced sensors, in the presence of uncorrelated sources and spatially white additive noise. Most high resolution techniques are developped in this context and are quite sensitive to discrepancies with respect to these assumptions. We propose an approach that has performances that are similar to those of the HR techniques but that can handle arbitrary arrays (with known geometry) and arbitrary (unknown) noise characteristics. We only consider the case of uncorrelated sources.

Among the methods that allow to handle arbitrary unknown noise one can distinguish those that use an unique parametric model [2], [3] (valid over the whole horizon) for the noise (a spatial AR model in general) and those that work on small spatial sectors [1] and adapt a different simple noise model in each sector (the local contribution of the noise is modeled by a constant or a slope in the local estimate of the spectrum). The first approaches only apply to linear equispaced arrays while the second, working locally, have difficulties with even slightly resonant spectra. Both approaches are computationally intensive.

\section{THE PROPOSED APPROACH}

The approach we propose is easy to implement and has an extremely reasonable computation cost. After a modeling step, performed once for ever for a given array (geometry), it relies upon simple numerical algorithms and mainly upon a linear programming algorithm such as the simplex method available in any scientific program library. The recently developed interior point methods can also be used. We will consider for simplicity that the sources and the array are in the same plane and the sources are in the far-field. A source is then characterized by its power and bearing (or spatial frequency).

For the source localisation problem, we start evaluating the outputs of a standard beamformer (taking into account the array geometry in the definition of the steering vector) at $M$ equispaced spatial frequencies where $M$ is the number of degrees of freedom (d.o.f.) of the array (the number of real d.o.f. of the covariance matrix of the snapshots). The vector $\hat{v}$ containing these outputs and an estimate $\hat{\Sigma}$ of the covariance matrix $\Sigma$ of this vector (computed using the usual complex-gaussian-vector model for the snapshots) are the inputs to our algorithm. Note that for an equispaced linear array having $N$ sensors, $M$ is equal to $2 N-1$ and there is a slight loss of information here since the transformation going from the covariance matrix of the snapshots (a sufficient statistic) to the beams is not one-to-one.

We then propose to find a parsimonious representation of the observed vector $\hat{v}$ on a basis or the association of different bases. Basis stays here for a set or collection of functions. While the Fourier representation is well adapted when one seeks to represent sinusoids, in our case different bases are obviously better adapted.

\subsection{The modeling step}

To represent the source-contributions to the $v$-vector, we take the basis whose elements (the $x$-vectors below) are the outputs of the beamformer to isolated unit-power sources in a large number of directions (the index $l$ of the basis element $x_{l}$ is linked to the spatial frequency of the source that produces it). If we denote $d \theta(\nu)$ the steering vector associated with spatial frequency $\nu$, the beamformer-output at spatial frequency $f$ to an unique source at frequency $\nu$ is simply :

$$
F_{0}(f)=\frac{1}{N^{2}}\left|d \theta(f)^{*} d \theta(\nu)\right|^{2}
$$

where $N$ denotes the number of sensors. For a linear equispaced array, the beamformer-output is the discrete normalized and sampled Fejer kernel.

To model the unknown noise contribution a specific basis is associated to this first one. Let $b_{k}$ denote an element of this basis. Since rational spectral densities allow to approximate any spectral density and since in some sense any rational density can be modeled as the sum of spectral 
densities associated with essentially $\mathrm{AR}(1)$ and $\mathrm{AR}(2)$ (or $\operatorname{ARMA}(2,1))$ processes, we built the $b_{k}$ elements as the outputs of the beamformer to a wide variety of spatial AR(1) and AR(2)-models. We typically parametrize the AR-model by their roots (poles) and consider an uniform grid in the magnitude-phase plane. To fix ideas, for the real AR(2)part of this basis, we take five different magnitudes (which characterize the damping factor) and sixteen different equispaced phases in the simulations below.

Finally, in order to be able to take into account the estimation error present in $\hat{v}$ because only a finite number of snapshots is available, one can adjoin a further basis to the two previous ones. The elements $\left\{\epsilon_{j}\right\}$ of this basis can be build using information coming from the covariance matrix $\Sigma$ of $\hat{v}$. Each element $\left\{\epsilon_{j}\right\}$ can for instance be the contribution to $\hat{v}$ of one realization of the estimation error.

We then seek a decomposition of $\hat{v}$ on these bases.

$$
\sum_{l} \alpha_{l} x_{l}+\sum_{k} \beta_{k} b_{k}+\sum_{j} \gamma_{j} \epsilon_{j}=\hat{v}
$$

Obviously such a decomposition is far from unique since we merged several different bases and some elements of a basis do have representations in terms of other elements. To single out one representation and possibly the true one, we need to define a criterion and an associated optimisation procedure. The basic idea is that the true representation is the sparsest one, the one with the fewest significant weights.

\subsection{The optimisation step}

Since we reconstruct an observed positive spectrum from positive elementary spectra, we consider only those decomposition having non-negative weights. To guarantee the parsimony we seek, we minimize the $\ell_{1}$ norm of the weights. To potentially obtain even sparser solutions, one could consider minimizing the $\ell_{p}$ quasi-norm of the weights for $0<p<1$ but the optimization problem then becomes non-convex and no straightforward algorithms are available as opposed to the $\ell_{1}$ norm for which the optimum is simply obtained as the solution of a linear program :

$$
\begin{aligned}
& \operatorname{Min} \sum_{l=1}^{L} \alpha_{l}+\sum_{k=1}^{K} \beta_{k}+\sum_{j=1}^{J} \gamma_{j} \\
& \text { under } \sum_{l} \alpha_{l} x_{l}+\sum_{k} \beta_{k} b_{k}+\sum_{j} \gamma_{j} \epsilon_{j}=\hat{v}
\end{aligned}
$$

$$
\alpha_{l} \geq 0, \quad \beta_{k} \geq 0, \quad \gamma_{j} \geq 0
$$

The theory behind linear programming is well established, let us simply say that there are no local minima and that if the global minimum is not an isolated point, all global minima belong to a convex set. Moreover the (global) minimum is always attained at an extreme point of the domain of solutions indicating that at most $M$ components of the solution ( $M$ weights) will be strictly positive at the optimum. This clearly guarantees some parsimony and is, besides the fact that algorithms solving LP's are easily available, the major reason for choosing this approach.

Another way to take into account the estimation errors is to use $\hat{\Sigma}$ to whiten the observation vector $\hat{v}$. We define $\hat{\tilde{v}}=$ $\hat{\Sigma}^{-1 / 2} \hat{v}$ and change accordingly the formulation of the linear program. We drop the columns $\left\{\epsilon_{j}\right\}$ and apply the same transformation $\hat{\Sigma}^{-1 / 2}$ to the remaining bases elements. We further replace the equality sign in the linear constraint (2) by upper and lower bounds. The linear program to be solved now is :

$$
\begin{array}{ll} 
& \operatorname{Min} \sum_{l} \alpha_{l}+\sum_{k} \beta_{k} \\
\text { under } \quad \hat{\tilde{v}}-\delta \boldsymbol{I} \leq \sum_{l} \alpha_{l} \tilde{x}_{l}+\sum_{k} \beta_{k} \tilde{b}_{k} \leq \hat{\tilde{v}}+\delta \boldsymbol{I} \\
\\
\alpha_{l} \geq 0, \quad \beta_{k} \geq 0,
\end{array}
$$

where $\boldsymbol{I}$ denotes a column vector of one's. The idea behind this last modification is the following. Since $\hat{v}$ (and $\hat{\tilde{v}})$ is corrupted by estimation errors there is no reason to ask the reconstructed model to fit exactly these observations, it is more adequate to ask the reconstructed model to pass within e.g. one standard deviation of the observations. Putting such a tube around the whitened observations is an easy way to take into account the estimation errors and is the one that has been used in the simulations below.

\section{ANALYSIS}

There are a number of important theoretical issues one should answer to justify this approach which, looking at the simulation results, has quite remarkable results.

The bases we introduced are redundant and as already indicated the solution is thus far from being unique. This non-uniqueness is important and indeed necessary. To solve two closely spaced sources the discretisation step in the spatial frequency domain has to be small and the number $L$ of elements must thus be large meaning that the redundancy is unavoidable. Provided the bases are well chosen, one can however somehow expect that the true and exact representation is the most parsimonious.

The identifiability issue is particularly important. It is addressed in the Appendix. It requires that the dictionaries are built so that, in the absence of estimation errors, if $v$ the second member in relation (2) is exactly obtained as a positive linear combination of a number of basis elements smaller than $M$, this sought-for solution is also the unique optimal solution (or at least belongs to the convex set of optimal solutions) of the linear program.

Another important issue concerns the theoretical evaluation of the asymptotic performances of this kind of approach. In our context, if $T$ denotes the number of snapshots available and $N$ being the number of sensors, the variance achievable for the bearing estimates is of order $T^{-1} N^{-3}$ and one can expect that this approach can attain a similar efficiency [6]. Assuming that the modeling step has been successful and thus that the optimum given by the solution to (3) is generically in the neighbourhood of the true value, one is of course interested in the statistical properties of this procedure.

An important parameter in this respect is $\delta$, the radius of the tube in (3). Assume that the true scenario generating $\hat{v}$ and thus $\hat{\tilde{v}}$ can be exactly described by our bases i.e. there are no discretization bias or errors. We then want that, with probability close to one, this exact representation belongs 
to the solution set of our LP (3) despite the estimation errors. Since, asymptotically in $T$, the components of $\hat{\bar{v}}$ are samples from independent normalised gaussian random variables we have to take $\delta$ so that the probability that the supremum of the difference of these $M$ variables and their mean value be larger than $\delta$ in absolute value is close to zero. For independent gaussian random variables, it is easy to show that $\delta$ has then to be of the order of $\sqrt{\log M}$.

With probability close to one our vector of estimates, say $\hat{\theta}$, is then such that $|\tilde{v}(\hat{\theta})-\hat{\tilde{v}}| \leq \delta$, this implies, taking into account the fact that $\Sigma$ is of order $T^{-1}$, that $|v(\hat{\theta})-\hat{v}| \leq \delta / \sqrt{T}$ and the variance of $v(\hat{\theta})$ thus of order $\delta^{2} / T$. Working backwards, the value of $\delta$ can then further be tuned according to the order of magnitude of the asymptotic variance of the estimates one can expect (for instance $\left.T^{-1} N^{-3}\right)$.

This same $\delta$ parameter also plays an important role in the sparsity issue. Obviously if it is taken very large, solutions using just one column of $A$ i.e. with just one positive weight will exist, while if is taken very small the solutions, if any, will be non-degenerate i.e. will have $M$ positive weights. This indicates that the value of $\delta$ allows to tune the number of sources (positive weights) in the optimal solution. This is the case, indeed, and one imagines easily that the stability or robustness of the position of a source for $\delta$ varying within reasonable bounds may be a good criterion to decide between true and spurious sources. Much work remains to be done along these issues.

\section{A SIMULATION RESULT}

To convince the reader of the interest of the proposed approach, let us present a simulated example that follows the above philosophy. We consider a linear equispaced array having 16 sensors (the example is taken from the litterature [2], remember that we can consider any geometry). The number of snapshots is 300 . There are 3 sources (this number is unknown and knowing it would be quite useless in our approach) at spatial frequencies : $-.17, .3536$, .409 ( $-20,45$ and 55 degrees) and corresponding power, respectively, $-10 \mathrm{~dB},-8 \mathrm{~dB},-5 \mathrm{~dB}$. The additive noise is simulated using a real AR(2) model with parameters .4 and -.8 (a pair of quite resonant complex conjugate poles). The AR(2)-process variance is taken equal to 1 . This fixes the $S N R$ which can be assessed from the beamformer output in figure 3. For this array there are 31 d.o.f. so that all the basis vectors are of dimension 31 . The spatial frequencies were sampled at a 93 equispaced points ( 1 runs from 1 to 93 in (3)). In the figures, we represent -as a function of the spatial frequency- the beamformer output $\hat{v}$ and the 93 optimal values of the $\alpha_{l}$ 's given by the LP algorithm represented by the *'s.

First, we only simulate the AR(2) noise. We apply our algorithm to these source-less data. In figure 1, we represent the output of the beamformer and the output of the algorithm, as far as the source localisation is concerned. The $\alpha_{l}$ weights are lower limited to $10^{-2}=-20 d B$ to draw the figure. Two weights only are greater than $10^{-2}$ but are small enough not to be confused with a source since they lie below the detection threshold of the considered array.
This means that our approach makes no confusion between sources and a resonant $A R(2)$ spectrum.

We then simulate the 3 sources in additive $0 d B$ white noise. In figure 2, we represent the output of the beamformer and the result of the algorithm when applied to these data. It locates the 3 sources without difficulty and an additional very low one around $f=.27$.

In figure 3 , we represent the output of the BF and our algorithm to a simulation of the complete scenario : the 3 sources and the AR(2) noise. The 3 sources are located without any apparent bias and without getting misleaded by the unknown quite resonant $\mathrm{AR}(2)$-noise.

We should emphasize that strictly the same algorithm, with no intermediate tuning, has been applied to the 3 situations above.

\section{APPENDIX}

We investigate in this appendix the conditions on the elements of the dictionnaries under which there is identifiability of any scenario. The meaning of this term will become clear below. Our linear program (LP) is in standard form and can be rewritten as:

$$
\begin{array}{cc}
\min & \boldsymbol{I}^{T} x \\
\text { s.t. } & A x=b \\
& x \geq 0
\end{array}
$$

where $I$ denotes the column vector filled with $n$ one's. $A$ is an $(m, n)$ with $n>m$ and even typically $n \gg m$. Each column in $A$ is a element of a dictionary, we will denote them by $A_{i}$. We assume from the beginning that any subset of $m$ columns of $A$ is full rank. This precludes, for instance, ambiguous array geometries, a difficulty that cannot be handled by linear algebra.

Let us consider a second member $b$ obtained as a positive linear combination of a number $k \leq m$ of columns of $A$ :

$$
b=\sum_{i=1}^{k} y_{j_{i}} A_{j_{i}}
$$

In our context, $k$ will in general be much smaller than $m$. For simplicity, we assume that $j_{i}=i$ in the sequel. Decomposing then the matrix $A$ into two matrices $B$ and $N: A=\left[\begin{array}{ll}B & N\end{array}\right]$, and the $x$-vector correspondingly into $x_{B}$ and $x_{N}$, we rewritte the previous relation as $b=B y$ or else $b=A x_{0}=B y+N 0$ with 0 a column vector of $n-k$ zeros.

By identifiablity we then mean that the basic (and in general degenerate) admissible solution $x_{0}$ that perfectly describes the scenario is also the optimal solution to the LP (or at least belongs to the convex set of global minima). $A$ priori nothing guaranties this property and the elements of the dictonnaries have to be built to satisfy it.

In the case where $k=m$, i.e. when $x_{0}$ in a non-degenerate basic feasible solution the necessary and sufficient condition for this solution to be also optimal is that for any vector $n$ of $N$, one must have :

$$
1-\boldsymbol{I}^{T} B^{-1} n \geq 0
$$

An interpretation of this condition is as follows. Define $z$ as the solution (un-restricted in sign) of $B z=n$, then $z=B^{-1} n$, and the condition above becomes $I^{T} B^{-1} n=$ $I^{T} z=\sum z_{i} \leq 1$. All the columns $n \in N$ must be such 
that $n=\sum z_{i} B_{i}$ with $\sum z_{i} \leq 1$. From a geometrical point of view this means that all the vectors $n$ have to belong to the negative closed half-space defined by the hyperplane $\left\{y \mid y=\sum B_{i} y_{i}, \sum y_{i}=1\right\}$ or, in a more standard form for an hyperplane, by $\left\{y \mid a^{T} y=1, a^{T}=\boldsymbol{I}^{T} B^{-1}\right\}$. Since this is to hold for any choice of $b$ built as a positive linear combination of columns of $A$ it implies that all the columns vectors $A_{i}$ of $A$ have to belong to a same hyperplane (defined by any subset of $m$ of them).

This same conclusion is arrived to if one seeks an analoguous condition for $k=1$ above i.e. when $\mathrm{b}$ is simply any column $A_{i}$ of $A$. One then wishes that $B y=b$ implies $\boldsymbol{I}^{T} y \geq 1$. Again, since this has to hold for any choice of $b=\overline{A_{i}}$ and $B$ a square submatrix of $A$ it implies that all the columns of $A$ have to belong to a same hyperplane.

One can, for instance, choose the columns to satisfy : $I^{T} A_{i}=1$ or else $e_{1}^{T} A_{i}=1$ with $e_{1}$ the first column of the identity matrix.

Unfortunatly, if the $A$ matrix satisfies this condition, one easily checks that all the admissible solutions have the same cost. This is, of course, a bad situation. Since it means that no optimization has to be performed and the simplex algorithms simply searches for a feasible point which has no reason to be close to the true (quite degenerate) sought-for solution unless it is the unique feasible point which is the case in the Pisarenko-Caratheodory situation [5].

In practice, the linear program we solve (3), is fortunatly not the one described above. Instead of imposing $A x=b$, we replace this equality constraint by $b-\delta \boldsymbol{I} \leq A x \leq b+\delta \boldsymbol{I}$ to allow for estimation errors. Then, even if the columns of $A$ belong to the same hyperplane, the feasible solutions have not the same cost. Performing an analoguous investigation as above for this new formulation has little meaning unless one introduces a probabilistic model for the estimation errors. Indeed, in the absence of estimation errors, the exact solution passes exactly through the middle of the tube and obviously does not take advantage of the flexibility it offers as does the optimal solution which is thus a biased estimate

Note that one can further decide to minimize only the $\ell_{1}$ norm of part of the unknowns, for instance the $\alpha_{l}$ 's.

\section{REFERENCES}

[1] P. Forster, G. Vezzosi. Application of spheroidal sequences to array processing. In Proc. ICASSP, pages 2268-2271, 1987.

[2] J.P. Le Cadre. Parametric methods for spatial signal processing in the presence of unknown colored noise fields. IEEE Transactions on ASSP, 37(7), 965-983, July 1989.

[3] A.H. Tewfik. Direction finding in the presence of colored noise by candidate identification. IEEE Transactions on Signal Processing, 39(9), 1933-1942, sept. 1991.

[4] D. G. Luenberger. Introduction to linear and nonlinear programming. Addison Wesley, 1973.

[5] J.J. Fuchs. Extension of the Pisarenko method to sparse linear arrays. In Proc. ICASSP 1995, pp. 2100-2103.

[6] J.J. Fuchs and H. Chuberre. "A deconvolution approach to source localization". IEEE Trans. on Sig. Proc., 42(6), 1462-1470, June 1994

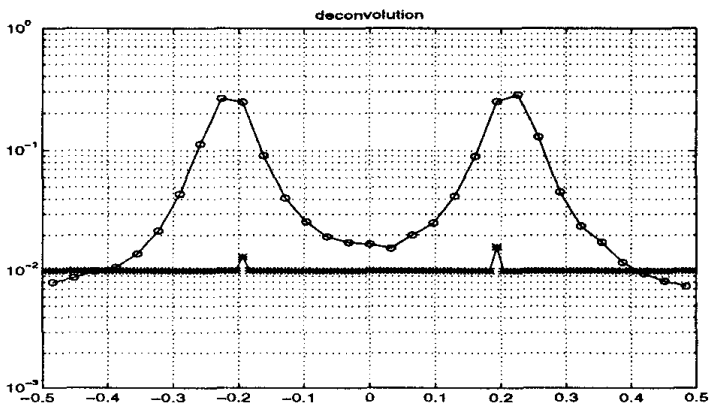

Figure 1. The real AR(2)-noise only. The algorithm does not confuse noise and sources, it detects two spurious sources of negligible amplitude.

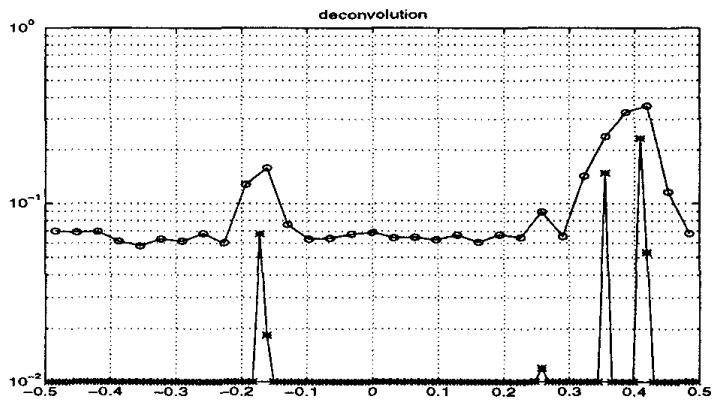

Figure 2. The 3 sources in white noise. The algorithm detects the 3 sources and does not confuse them with noise.

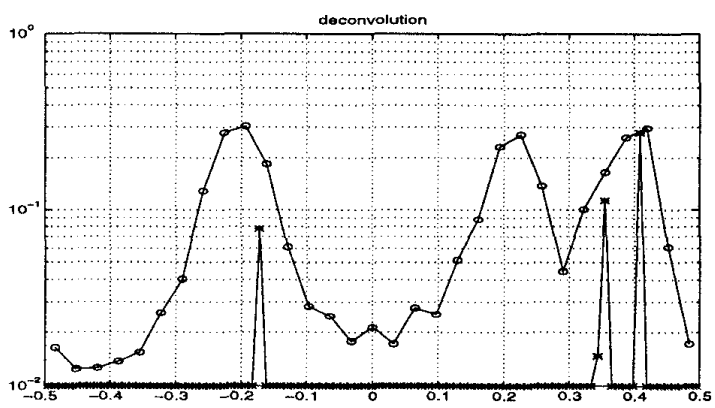

Figure 3. The complete scenario : the $\operatorname{AR}(2)$-noise and the 3 sources. The algorithm detects correctly the 3 sources despite the presence of a quite resonnant noise spectrum. 